\title{
A Comparison of Subconjunctival Wound Healing between Different Methods of Dissecting Subconjunctival Tissues
}

\author{
Ruowen Gong ${ }^{a}, b \quad$ Chengguo Zuo ${ }^{a} \quad K^{2}$ ling Wu ${ }^{a}$ Simin Zhang ${ }^{a} \quad X_{i}$ Qin $^{a}$ \\ Yangyang $\mathrm{Li}^{\mathrm{a}}$ Xinbo Gao ${ }^{\mathrm{a}}$ Danping Huang ${ }^{\mathrm{a}}$ Mingkai Lin ${ }^{\mathrm{a}}$ \\ aState Key Laboratory of Ophthalmology, Zhongshan Ophthalmic Center, Sun Yat-sen University, \\ Guangzhou, China; 'bepartment of Ophthalmology, Eye and ENT Hospital of Fudan University, Shanghai, China
}

\section{Keywords}

Glaucoma filtration surgery $\cdot$ Wound healing $\cdot$ Conjunctival flap · Tenonectomy

\begin{abstract}
Objective: To compare different methods for dissecting subconjunctival tissues by developing subconjunctival wound healing models. Methods: New Zealand white rabbits were separated into 3 groups based on the method by which the rabbit subconjunctival wound healing model was generated: subconjunctival tissues were dissected episclerally (EPI) or subepithelially (SUB), with a corresponding blank control (CON). All the cases in the experimental groups were surgically prepared with conjunctival flaps, and they were sacrificed on the third postoperative day. At the surgical sites, the protein levels of hypoxia-inducible factor-1 (HIF-1)-a, vascular endothelial growth factor (VEGF)-A, and matrix metalloproteinase (MMP)-2 were detected by Western blot, morphological vascularity was measured by Adobe Photoshop, and subconjunctival fibrosis was assessed by histology. $\boldsymbol{R e}$ sults: Compared with the CON group, both the EPI and SUB groups showed significantly upregulated protein levels of HIF-1a, VEGF-A, and MMP-2. In addition, the protein levels of
\end{abstract}

HIF-1a, VEGF-A, and MMP-2 were higher in the EPI group than in the SUB group. Morphological vascularity was significantly elevated in the EPI group compared with the SUB and CON groups. Collagen content was markedly increased in the EPI group compared with the SUB and CON groups. Conclusions: Dissecting subconjunctival tissues subepithelially inhibits subconjunctival fibrosis, which may be instructive in tenonectomy in filtration surgery.

(C) 2020 S. Karger AG, Basel

\section{Introduction}

Filtration surgery is one of the most common surgical treatments of glaucoma that filters aqueous humor into the subconjunctiva to form a filtering bleb. Nevertheless, filtration surgery still has complications that affect the success rate, and the most evident complication that causes filtration obstruction and even surgery failure is bleb scarring. Furthermore, the main reason for bleb scarring is the excessive proliferation of the fibroblasts of Tenon's capsule [1]. Therefore, to concentrate on subconjunctival scarring, we developed a subconjunctival wound healing model.
Mingkai Lin or Danping Huang

State Key Laboratory of Ophthalmology, Zhongshan Ophthalmic Center Sun Yat-sen University, 54 South Xianlie Road Guangzhou 510000 (China)

linmk@mail.sysu.edu.cn or hdanp@mail.sysu.edu.cn 


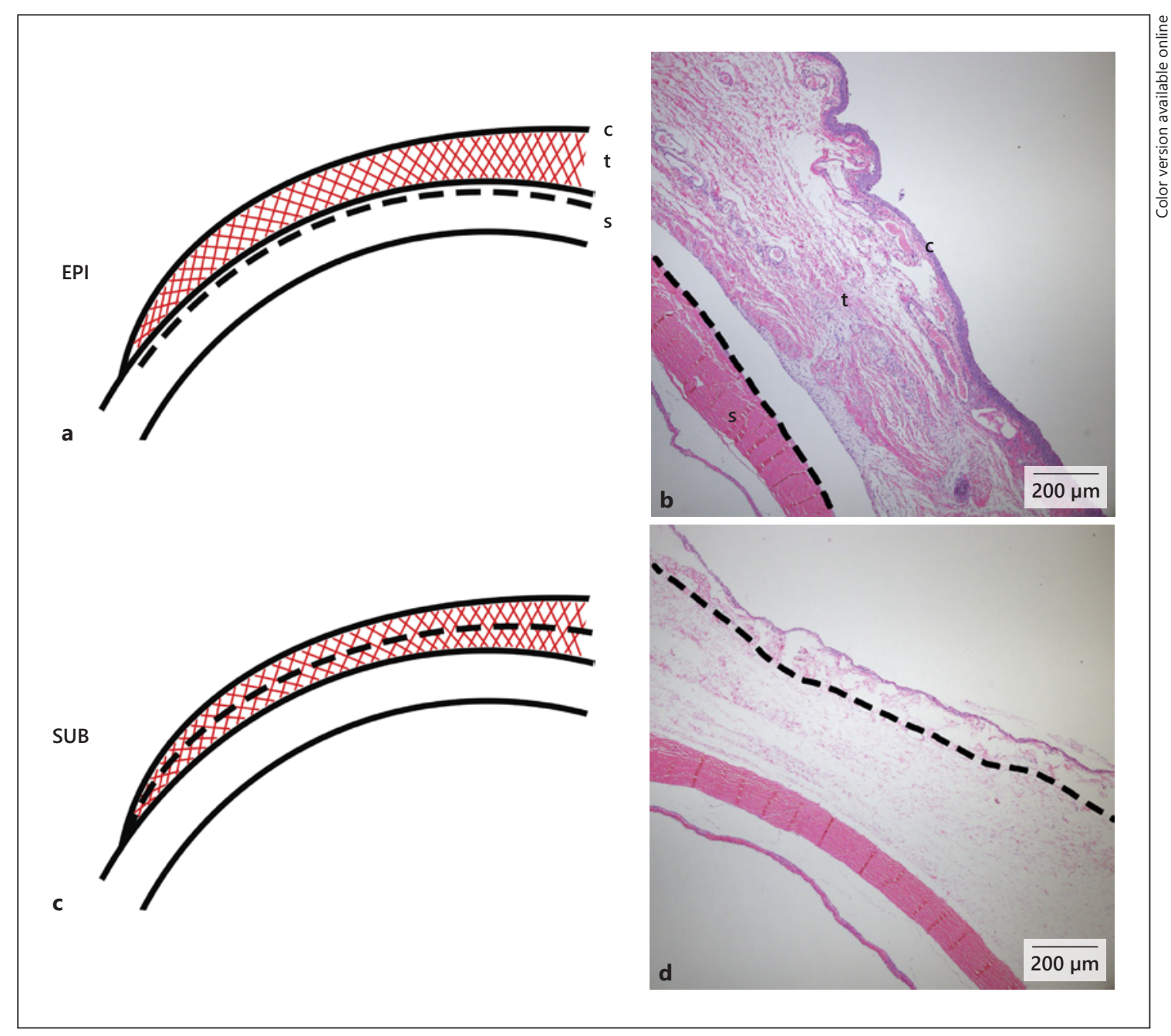

Fig. 1. a Dissecting subconjunctival tissues at different depths. Schematic depiction showing episcleral dissection of the subconjunctival tissues (Tenon's capsule and conjunctiva were dissected bluntly to the surface layer of the sclera). b Hematoxylin and eosin staining on the third day after episcleral dissection. c Schematic depiction showing the subepithelial dissection of the subconjunc-

Compared with conventional trabeculectomy, corneal trabeculectomy which is performed with less conjunctival incision causes less subconjunctival fibrosis [2]. Minimally invasive external mini-glaucoma shunt implantation with less conjunctival dissection potentially causes less conjunctival scarring [3]. Moreover, tenonectomy in trabeculectomy may help reduce fibrosis and therefore improves the success rate [4]. These results inspired us to explore whether dissecting subconjunctival tissues at different depths influences scar formation.

Additionally, many proteins that promote scar formation, including vascular endothelial growth factor (VEGF) tival tissues (blunt dissection was performed between Tenon's capsule tissues to avoid interfering with the sclera). d Hematoxylin and eosin staining on the third day after subepithelial dissection. The dotted line is equivalent to the position of the dissection. Scale bar, $200 \mu \mathrm{m}$. c, conjunctiva; t, Tenon's capsule; s, sclera; EPI, episcleral; SUB, subepithelial. and matrix metalloproteinases (MMPs), are involved in postoperative subconjunctival wound healing [5-8]. Moreover, hypoxia-inducible factor-1 (HIF-1)- $\alpha$ is the most potent regulator of oxygen homeostasis during tissue repair and wound healing $[9,10]$. Our previously published data [11] showed that HIF-1 $\alpha$ was expressed on rabbit conjunctiva and Tenon's capsule after developing a rabbit model of subconjunctival wound healing, and the upregulation of HIF-1 $\alpha$ was associated with subconjunctival fibrosis.

The purpose of this study was to dissect the subconjunctival tissues between Tenon's capsule tissues or to the 
surface layer of the sclera, in order to compare the effect of these procedures on postoperative subconjunctival fibrosis, morphological vascularity, and the proteins that promote scar formation, including HIF-1 $\alpha$, VEGF-A, and MMP-2, at the surgical sites.

\section{Materials and Methods}

\section{Animals}

All procedures involving animal experiments followed the ARVO Statement for the Use of Animals in Ophthalmic and Vision Research and were approved by the Animal Ethics Committee of Zhongshan Ophthalmic Center, Sun Yat-sen University. Female New Zealand white rabbits (12-14 weeks old, $2.0-3.0 \mathrm{~kg}$ ) were provided by the Animal Center of Zhongshan Ophthalmic Center, Sun Yat-sen University. All cases were randomly separated into 3 groups. The separation was made according to the methods for making conjunctival flaps at different depths: episcleral (EPI) or subepithelial (SUB) dissection of subconjunctival tissues, with a corresponding blank control group (CON).

\section{Surgical Procedure}

Animals were surgically prepared with conjunctival flaps to imitate the progression of subconjunctival wound healing. Pentobarbital sodium (40 mg/kg; Sigma, Santa Clara, CA, USA) was injected intramuscularly to induce general anesthesia, and proxymetacaine hydrochloride eye drops (Alcon, Fort Worth, TX, USA) were used to induce local anesthesia. First, each rabbit eye in the experimental groups was prepared with a limbus-based conjunctival flap $5 \times 4 \mathrm{~mm}$ in size, and Tenon's capsule and the conjunctiva were dissected episclerally (Tenon's capsule and conjunctiva were dissected bluntly to the surface layer of the sclera) or subepithelially (blunt dissection was performed between Tenon's capsule tissues to avoid interfering with the sclera) (Fig. 1); the remaining eyes that did not undergo surgery were assigned to the CON group. No antiscarring or anti-inflammatory medicine was used. Finally, the conjunctiva was closed with 10-0 nylon suture (Alcon). Subconjunctival fibrosis was significantly evident on the third postoperative day based on our previous study [11]. Therefore, all rabbits were sacrificed on the third day after surgery.

\section{Western Blot Analysis}

RIPA lysis buffer (Beyotime, Shanghai, China) and phenylmethanesulfonyl fluoride (Beyotime) were combined 100:1 to extract total protein from the subconjunctival tissue at the surgical sites. After centrifuging all samples at $14,000 \mathrm{~g}$ and $4^{\circ} \mathrm{C}$ for $5 \mathrm{~min}$ to obtain protein supernatant, the samples were denatured with SDS-PAGE Sample Loading Buffer, $5 \mathrm{X}$ (Beyotime) at $100^{\circ} \mathrm{C}$ for 5 min. Then, samples were stored at $-20^{\circ} \mathrm{C}$ until use. The samples were separated electrophoretically (Bio-Rad, Hercules, CA, USA) in 8 or $12 \%$ SDS-PAGE gels (Beyotime), and the proteins were transferred (Bio-Rad) to a polyvinylidene fluoride membrane $(0.45 \mu \mathrm{m}$; Millipore, Billerica, USA). After blocking in Tris-buffered saline containing $0.1 \%$ Tween-20 and 5\% dry milk for $1 \mathrm{~h}$ at room temperature, the blots were probed with specific antibodies overnight at $4^{\circ} \mathrm{C}$ : a monoclonal mouse anti-HIF-1 $\alpha$ antibody (NB100-105; Novus, Colorado, USA) diluted 1:250, a monoclonal

Different Dissections of Subconjunctival

Tissues mouse anti-VEGF-A antibody (ab1316; Abcam, Cambridge, UK) diluted 1:200, a monoclonal mouse anti-MMP-2 antibody (ab2462; Abcam) diluted 1:50, or a polyclonal rabbit anti- $\beta$-actin antibody (4970; Cell Signaling Technology, Boston, MA, USA) diluted $1: 1,000$. Then, the blots were incubated with the respective secondary antibodies (Cell Signaling Technology) for $1 \mathrm{~h}$ at room temperature. The specific bands were visualized via a Bio-Rad Chemi Doc XRS imaging system (Bio-Rad) using Immobilon Western Chemiluminescent HRP Substrate (Millipore). Imaging with ImageJ software (Rawak Software Inc., Stuttgart, Germany) was performed to analyze the bands, and the protein levels of HIF-1a, VEGF-A, and MMP-2 were expressed as ratios to $\beta$-actin levels. All samples were randomly divided into 3 groups based on surgical methods (6 in each group).

Evaluation of Morphological Characteristics at Surgical Sites

The appearance of the surgical area was observed on the third postoperative day to evaluate morphological characteristics. Postoperative images were acquired by an operating microscope (Leica M844, Wetzlar, Germany). Morphological vascularity at the surgical site was measured using Adobe Photoshop CC (Adobe Systems Incorporated, San Jose, CA, USA), and it was interpreted as the ratio of the vascular area to the total surgical area. All samples were randomly separated into 3 groups based on surgical methods ( 3 in each group).

\section{Histology}

The surgically impacted regions of the rabbit eyes were collected, immediately fixed in $4 \%$ paraformaldehyde overnight at room temperature, and embedded in paraffin wax. Tissues were sectioned at $5-\mu \mathrm{m}$ thickness. Once the resulting sections were deparaffinized, they were stained with hematoxylin and eosin or Masson's trichrome. The slides were analyzed via light microscopy. Additionally, the slides stained with Masson's trichrome were observed to assess collagen content. According to different surgical methods, all samples were randomly divided into 3 groups (3 in each group).

\section{Statistical Analysis}

Data were analyzed using SPSS software (version 24.0, IBM SPSS Statistics; IBM Corporation, Chicago, IL, USA), and the results are presented as mean $\pm \mathrm{SD}$. For 3 groups, one-way analysis of variance was used. Further multiple comparisons were performed with the LSD- $t$ test. Pearson's coefficient was applied for bivariate correlations. For all tests, statistical analyses were two tailed, and $p$ values $<0.05$ indicated statistical significance.

\section{Results}

The Protein Levels of HIF-1 $\alpha, V E G F-A$, and MMP-2

As shown in Figure $2 \mathrm{a}$ and d, the protein levels of HIF$1 a$ were significantly increased in the EPI $(p<0.001)$ and SUB $(p<0.01)$ groups compared with the CON group. Further, the protein level of HIF-1 $\alpha$ was higher in the EPI group than in the SUB group $(p<0.05)$. Additionally, both the EPI ( $p<0.001$ and $p<0.001$, respectively) and 


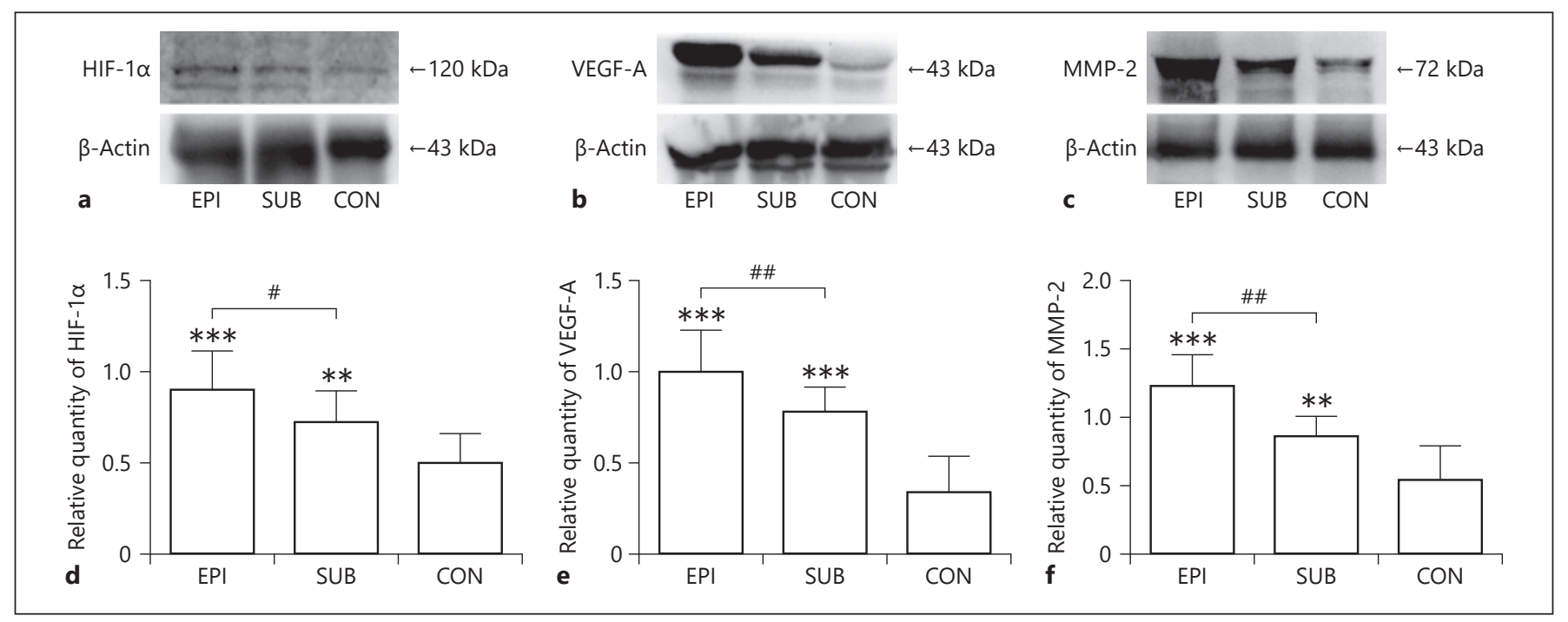

Fig. 2. The protein levels of HIF-1a in the EPI, SUB, and CON groups (a); the protein level of VEGF-A in the EPI, SUB, and CON groups (b); the protein level of MMP-2 in the EPI, SUB, and CON groups (c); analysis of the gray value of the HIF-1 $\alpha$ band (d); analysis of the gray value of the VEGF-A band (e); analysis of the gray value of the MMP-2 band (f). $n=6$ for each group. Compared with
CON, ${ }^{* * *} p<0.001$ and ${ }^{* *} p<0.01$; comparisons between EPI and SUB, ${ }^{\# \#} p<0.01$ and ${ }^{\#} p<0.05$. EPI, episcleral; SUB, subepithelial; CON, control; HIF-1 $\alpha$, hypoxia-inducible factor-1 $\alpha$, VEGF-A, vascular endothelial growth factor-A; MMP-2, matrix metalloproteinase- 2 .
SUB $(p<0.001$ and $p<0.001$, respectively) groups showed upregulated protein levels of VEGF-A and MMP-2 compared with the CON group (Fig. 2b-f). In comparison with the SUB group, the EPI group showed increased protein levels of VEGF-A and MMP-2 $(p<0.01$ and $p<0.01$, respectively). Moreover, the protein level of HIF-1a was positively correlated with the protein levels of VEGF-A $(r=0.58 ; p<0.05)$ and MMP-2 $(r=0.73 ; p<0.01)$.

\section{Morphological Characteristics of the Surgical Sites}

Figure 3 depicts the appearance of the surgical sites. Compared with the CON group (Fig. 3c), dilated and tortuous vessels were more evident both in the EPI (Fig. 3a) and SUB (Fig. 3b) groups. Additionally, the morphological vascularity was more severe both in the EPI and SUB groups than in the CON group. Further, the morphological vascularity was significantly increased in the EPI group $(26.42 \pm 8.12[\%])$ than in the SUB $(17.87 \pm 3.61$ $[\%], p<0.05)$ and CON $(12.60 \pm 2.11[\%], p<0.01)$ groups.

\section{Histological Characteristics of the Surgical Sites}

Hematoxylin and eosin staining displayed the structure of the conjunctiva and Tenon's tissues (Fig. 4a-c). Subepithelial connective tissues were loosely arranged in the CON (Fig. 4c) and SUB (Fig. 4b) groups but were densely arranged in the EPI group (Fig. 4a). Masson's trichrome staining revealed increased collagen content in the EPI group (Fig. 4d) compared with that in the SUB (Fig. 4e) and CON (Fig. 4f) groups.

\section{Discussion}

Glaucoma is the leading cause of irreversible blindness worldwide, and controlling intraocular pressure is the key to treating glaucoma. When medication and/or laser surgery have proven insufficient, glaucoma filtration surgery (GFS) remains the gold standard for controlling intraocular pressure $[12,13]$. Scar formation at the surgical site leads to the obstruction of aqueous flow and even filtration failure. Therefore, it is necessary to study postoperative scarring in animal models.

Animal models of postoperative scarring encompass the rabbit trabeculectomy model [14], the monkey trabeculectomy model [15], the rabbit tube drainage surgery model [12], the rat tube drainage surgery model [16], the rabbit full-thickness sclerectomy model [17], and the mouse conjunctival scarring model made by subconjunctival injection [18]. The physiology of monkeys is more similar to that of humans, in contrast to other animals, but their expense impedes their use in research. More- 


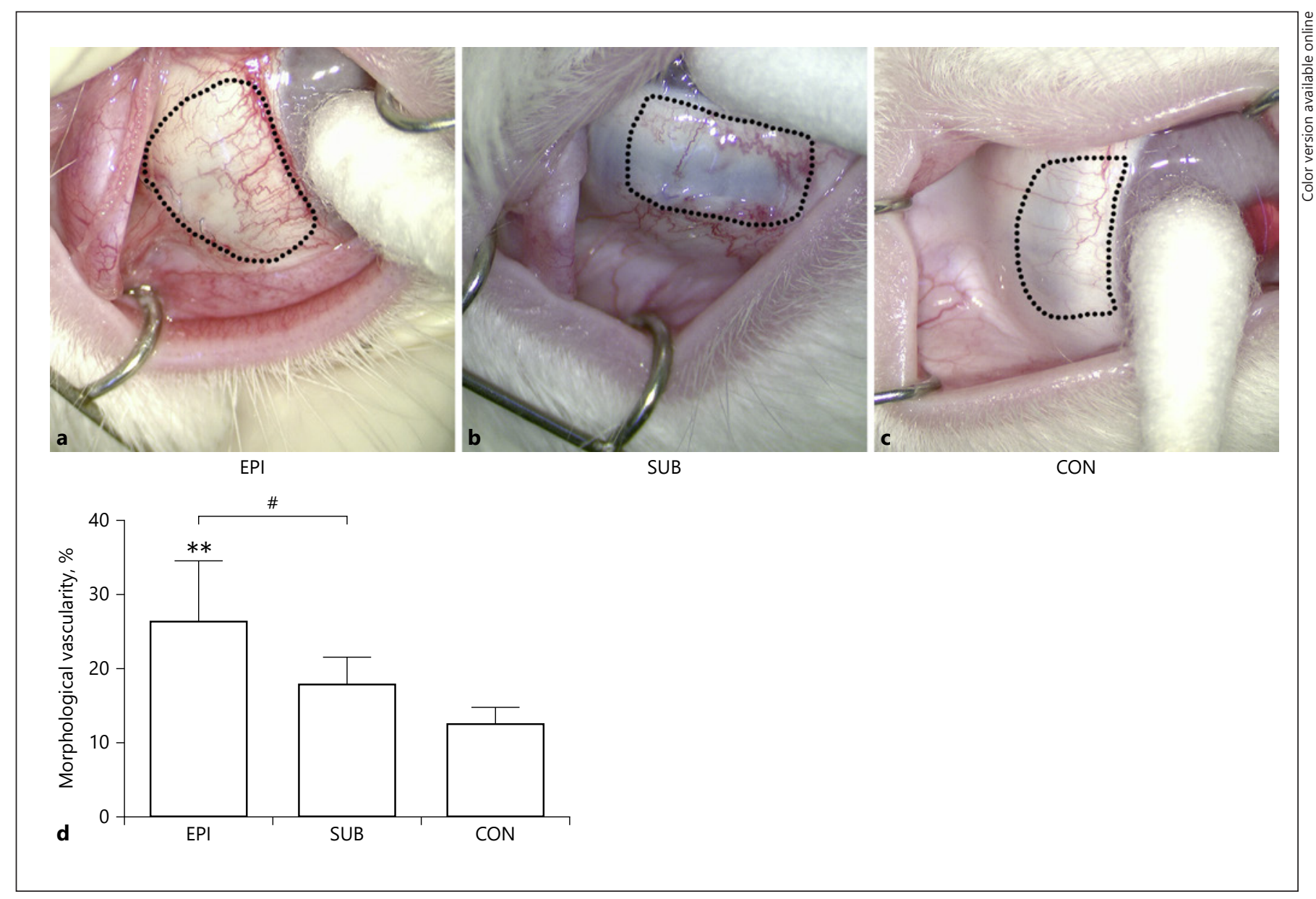

Fig. 3. a-c Representative images of the surgical sites. The morphological vascularity was more evident in the EPI group (a) than in the SUB (b) and CON groups (c). The surgical site is circumscribed by the dotted line. $\mathbf{d}$ Analysis of morphological vascularity. $n=3$ for each group. Compared with CON, ${ }^{* *} p<0.01$; comparisons between EPI and SUB, ${ }^{*} p<0.05$. EPI, episcleral; SUB, subepithelial; CON, control. over, studying postoperative scarring in a rat or mouse model is difficult, as their eyes are too small for the complicated surgeries. Due to the feasibility of performing the complicated surgery, we selected the rabbit for a postoperative scarring model. As the main reason for bleb scarring is the excessive proliferation of the fibroblasts of Tenon's capsule [1], we constructed a rabbit subconjunctival wound healing model to focus on subconjunctival scarring.

Studies concerning different methods for making conjunctival flaps include fornix- and limbal-based conjunctival flaps [19], different methods of suture [20], design of radial conjunctival incision [21], and partial or complete tenonectomy [22]. There has been no research on different methods for dissecting subconjunctival tissues. A re- cent report showed that minimally invasive external mini-glaucoma shunt implantation with less conjunctival dissection had the potential for less conjunctival scarring [3], and a previous study indicated that corneal trabeculectomy had less subconjunctival scarring than conventional trabeculectomy [2]. Trabeculectomy with radial conjunctival incision restricted bleb formation compared with trabeculectomy without radial conjunctival incision [21]. Therefore, these studies showed the potential influence on scar formation by interfering with conjunctiva and Tenon's capsule and inspired us to study whether dissecting subconjunctival tissues at different depths influences subconjunctival fibrosis. Furthermore, excision of Tenon's capsule during trabeculectomy helps in preventing fibrosis and enhances the chances of success [4]. Ten- 

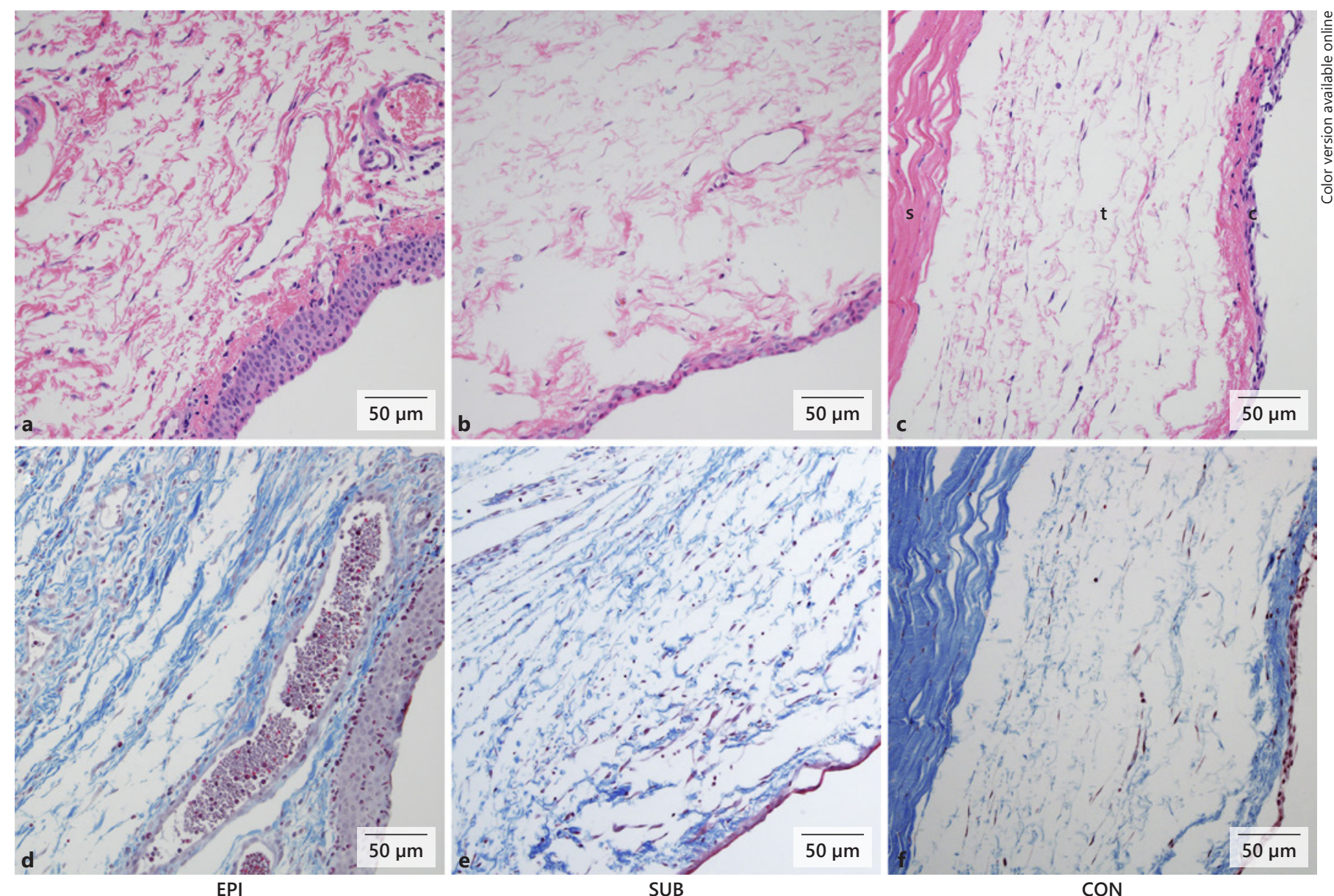

Fig. 4. Hematoxylin and eosin staining displayed the subconjunctival structure. Subepithelial connective tissues were densely arranged in the EPI group (a) and loosely arranged in the SUB (b) and CON groups (c). Masson's trichrome staining revealed that the collagen content at the surgical site markedly increased in the

EPI group (d), while there was no evident increase in collagen in the SUB group (e) compared to that in the CON group (f). Scale bar, $50 \mu \mathrm{m} . n=3$ for each group. $\mathrm{c}$, conjunctival epithelium; $\mathrm{t}$, Tenon's capsule; s, sclera; EPI, episcleral; SUB, subepithelial; CON, control.

on's capsule needs to be excised during filtration surgery to alleviate fibroblastic response, especially for the thick and active Tenon's capsule [23]. Tenonectomy can be made from Tenon's capsule to Tenon's capsule or to the surface layer of the sclera. However, there has been no study concerning whether tenonectomy at the sclera influences fibrosis. To our knowledge, this is the first study to explore the impact of interfering with the sclera during dissection of subconjunctival tissues on subconjunctival wound healing.

It has been reported that oxygen availability during tissue repair was the lowest on the third day during wound healing [9], and HIF-1 $\alpha$ is the most sensitive factor to hypoxia during wound healing $[9,10]$. Our previous data showed that HIF-1 $\alpha$ peaked on the third postoperative day in a rabbit subconjunctival wound healing model [11], suggesting that HIF-1 $\alpha$ can be activated at an early phase of subconjunctival scarring. Additionally, the activated transcript expression of some growth factors, cytokines, and chemokines at an early stage after GFS was corroborated [24]. Hence, we detected the levels of proteins that promote scar formation on the third day after surgery. In the present study, we dissected subconjunctival tissues episclerally or subepithelially and sacrificed rabbits on the third postoperative day to examine the expression of proteins promoting scar formation, including HIF-1 $\alpha$, VEGF-A, and MMP-2, at the surgical sites. HIF$1 \alpha$, VEGF-A, and MMP-2 were significantly upregulated in the EPI group compared with the SUB group. To begin with, we speculate that the significantly upregulated pro- 
tein levels of HIF-1 $\alpha$ in the EPI group stem from the more extensive damage of Tenon's tissues, which not only results in more extensive disruption of the vasculature that damages oxygen delivery but also promotes the recruitment of inflammatory cells at the surgical site that exacerbate oxygen consumption. Under hypoxia, HIF-1a ubiquitination by a Von Hippel-Lindau ubiquitin-protein ligase complex followed by proteosomal degradation is inhibited [10], and the active transcription factor HIF1 is then formed due to the combination of the stabilized HIF- $1 \alpha$ subunit and the HIF- $1 \beta$ subunit [10], which may promote expression of downstream target genes to accelerate the subsequent progression of subconjunctival wound healing. Studies [25-28] have documented that the stabilization of HIF- $1 \alpha$ accelerates wound healing, and, conversely, the loss of HIF- $1 \alpha$ leads to slower cutaneous wound healing. HIF-1 $\alpha$ has been considered to be an important determinant of healing outcomes $[9,10]$, but the impact of HIF-1a on subconjunctival wound healing is unknown.

Moreover, we attribute the significantly upregulated protein level of VEGF-A in the EPI group to the deeper subconjunctival dissection, which caused more extensive damage to Tenon's tissues. The severe disruption of the vasculature promotes the influx of vessel-derived cytokines, inflammatory cells, and growth factors, such as VEGF-A. To maintain the integrity of the vessels, more activated platelets release a wide array of growth factors, such as VEGF-A [29]. As a proangiogenic mediator, VEGF-A results in angiogenesis in wound repair [30]. More importantly, VEGF-A acts as a profibrotic mediator, by indirect effects on stimulating endothelial cells or amplifying inflammation and possible direct effects on fibroblasts, to promote scar formation [31]. Additionally, it was demonstrated that at the early "acute inflammatory" phase in the wound healing process after GFS, VEGFA expression increased significantly at both the transcript and protein levels [24], which is consistent with what we found in the present study. Consequently, the elevated secretion of VEGF-A in the EPI group promotes subconjunctival wound healing.

Apart from the higher protein level of VEGF-A, the significantly increased protein level of MMP-2 in the EPI group can also be explained by the more extensive damage to Tenon's tissues. Extensive tissue damage intensifies inflammation, activates fibroblasts, and accelerates neutrophil and macrophage recruitment, which subsequently gives rise to greater secretion of MMP-2. In addition, the enhanced expression of endothelial cells elevates the secretion of MMP-2 [32]. MMP-2 was highly expressed

Different Dissections of Subconjunctival Tissues in the wound area after GFS [7]. Moreover, interfering with MMP-2 using exogenous tissue inhibitor of metalloproteinase-2 delayed filtering bleb scarring [33], suggesting that the imbalance between MMP-2 and exogenous tissue inhibitor of metalloproteinase- 2 may play an indispensable role in subconjunctival wound healing. As a consequence, the increased MMP-2 expression in the EPI group degrades the extracellular matrix selectively and promotes fibrosis [29].

Our study also showed that the protein levels of VEGFA and MMP-2 were positively correlated with the protein level of HIF-1a. We speculate that dissection of subconjunctival tissues could disrupt the vasculature and cause inflammation, which exacerbates hypoxia and ischemia, elevates the protein level of HIF- $1 \alpha$, and then initiates the expression of its potential downstream target genes [10, 30], such as VEGF-A and MMP-2. However, this remains to be explored in depth.

Morphological vascularity is a crucial element in the evaluation of postoperative filtering blebs [34-36]. Increased vascularity was observed in the scarring filtering blebs [5]. Thus, the level of rabbit conjunctival vascularity was associated with subconjunctival fibrosis to some extent. Our study indicated that the level of subconjunctival vascularity was more severe in the EPI group than in the SUB group, which can be explained by the greater subconjunctival fibrosis in the EPI group as follows.

Compared with the SUB and CON groups, the EPI group showed increased collagen content. A study demonstrated the increased collagen synthesis of keloid fibroblasts under hypoxia in contrast to that of keloid fibroblasts under normoxia [37]. As the most sensitive factor to hypoxia, HIF-1a contributed to the establishment of fibrosis [38] and was considered to be an important determinant modulating wound healing $[9,10]$. Moreover, our previous studies showed that the downregulation of HIF-1 $\alpha$ alleviated angiogenesis $[39,40]$. As a result, we speculate that the deeper subconjunctival dissection in the EPI group could cause more severe disruption of the vasculature and aggravate inflammation, which would exacerbate hypoxia and ischemia, consequently elevate the expression levels of HIF-1, VEGF, MMPs, and other proteins that promote scar formation, and eventually accelerate subconjunctival angiogenesis and subconjunctival fibrosis. Therefore, the subepithelial dissection of subconjunctival tissues could be beneficial to the inhibition of subconjunctival fibrosis.

This study still has limitation. This is a rabbit model of conjunctival scarring, which cannot exactly reflect subconjunctival wound healing in human eyes. Whether the 
conclusions can be applied in humans will require further investigation.

In conclusion, compared with subepithelial dissection of rabbit subconjunctival tissues, episcleral dissection resulted in higher levels of cytokines and growth factors that accelerated healing. Hence, subepithelial dissection of subconjunctival tissues could be a more appropriate way to reduce subconjunctival fibrosis and may be instructive in the excision of Tenon's capsule in trabeculectomy.

\section{Statement of Ethics}

All procedures concerning animal experiments followed the ARVO Statement for the Use of Animals in Ophthalmic and Vision Research and were approved by the Animal Ethics Committee of Zhongshan Ophthalmic Center, Sun Yat-sen University.

\section{Conflict of Interest Statement}

All authors of this publication have no conflicts of interest to declare.

\section{Funding Sources}

This study was supported by the National Natural Science Foundation of China (81570846 and 81970808) and the Natural Science Foundation of Guangdong Province (2019A1515011196).

\section{Author Contributions}

M.L. and D.H. contributed to designing the work; R.G., C.Z., and K.W. contributed to conducting the work; and S.Z., X.Q., Y.L., and X.G. contributed to revising the manuscript.

\section{References}

1 Lim DH, Kim TE, Kee C. Evaluation of adenovirus-mediated down-regulation of connective tissue growth factor on postoperative wound healing after experimental glaucoma surgery. Curr Eye Res. 2016;41(7):951-6.

2 Cioffi GA, Van Buskirk EM. Corneal trabeculectomy without conjunctival incision. Extended follow-up and histologic findings. Ophthalmology. 1993;100(7):1077-82.

3 Hoffman RS, Crandall AS, Crandall DA, Fine $\mathrm{IH}$, Packer M, Sims AC. Minimally invasive external mini-glaucoma shunt implantation without conjunctival dissection. J Glaucoma. 2014;23(4):254-7.

4 Awadein A, El Sayed YM. Excision of Tenon capsule in pediatric trabeculectomy: a controlled study. J Glaucoma. 2016;25(1):39-44.

5 Schlunck G, Meyer-ter-Vehn T, Klink T, Grehn F. Conjunctival fibrosis following filtering glaucoma surgery. Exp Eye Res. 2016; 142:76-82.

6 Li Z, Van Bergen T, Van de Veire S, Van de Vel I, Moreau H, Dewerchin M, et al. Inhibition of vascular endothelial growth factor reduces scar formation after glaucoma filtration surgery. Invest Ophthalmol Vis Sci. 2009; 50(11):5217-25.

7 Shima I, Katsuda S, Ueda Y, Takahashi N, Sasaki $\mathrm{H}$. Expression of matrix metalloproteinases in wound healing after glaucoma filtration surgery in rabbits. Ophthalmic Res. 2007; 39(6):315-24.

8 Wong TT, Mead AL, Khaw PT. Matrix metalloproteinase inhibition modulates postoperative scarring after experimental glaucoma filtration surgery. Invest Ophthalmol Vis Sci. 2003;44(3):1097-103.

9 Nauta TD, van Hinsbergh VW, Koolwijk P. Hypoxic signaling during tissue repair and regenerative medicine. Int J Mol Sci. 2014; 15(11):19791-815.
10 Hong WX, Hu MS, Esquivel M, Liang GY, Rennert RC, McArdle A, et al. The role of hypoxia-inducible factor in wound healing. Adv Wound Care. 2014;3(5):390-9.

11 Gong R, Wu K, Zuo C, Huang D, Lin M. Expression of hypoxia inducible factor-1HIFlin a rabbit model of conjunctival wound healing. J Sun Yat-sen Univ (Med Sci). 2018; 39(6):827-34

12 Lukowski ZL, Min J, Beattie AR, Meyers CA, Levine MA, Stoller G, et al. Prevention of ocular scarring after glaucoma filtering surgery using the monoclonal antibody LT1009 (sonepcizumab) in a rabbit model. J Glaucoma. 2013;22(2):145-51.

13 Min J, Lukowski ZL, Levine MA, Meyers CA, Beattie AR, Schultz GS, et al. Prevention of ocular scarring post glaucoma filtration surgery using the inflammatory cell and platelet binding modulator saratin in a rabbit model. PLoS One. 2012;7(4):e35627.

14 Zhong H, Sun G, Lin X, Wu K, Yu M. Evaluation of pirfenidone as a new postoperative antiscarring agent in experimental glaucoma surgery. Invest Ophthalmol Vis Sci. 2011; 52(6):3136-42.

15 Ye H, Qian Y, Lin M, Duan Y, Sun X, Zhuo Y, et al. Cationic nano-copolymers mediated IKK $\beta$ targeting siRNA to modulate wound healing in a monkey model of glaucoma filtration surgery. Mol Vis. 2010;16:2502-10.

16 Sherwood MB, Esson DW, Neelakantan A, Samuelson DA. A new model of glaucoma filtering surgery in the rat. J Glaucoma. 2004; 13(5):407-12.

17 Trope GE, Cheng YL, Sheardown H, Liu GS, Menon IA, Heathcote JG, et al. Depot drug delivery system for 5 -fluorouracil after filtration surgery in the rabbit. Can J Ophthalmol. 1994;29(6):263-7.
18 Reichel MB, Cordeiro MF, Alexander RA, Cree IA, Bhattacharya SS, Khaw PT. New model of conjunctival scarring in the mouse eye. Br J Ophthalmol. 1998;82(9):1072-7.

19 Al-Haddad C, Abdulaal M, Al-Moujahed A, Ervin AM. Fornix-based versus limbal-based conjunctival trabeculectomy flaps for glaucoma. Cochrane Database Syst Rev. 2015; 11(11):CD009380.

20 Pfeiffer N, Grehn F. Improved suture for fornix-based conjunctival flap in filtering surgery. Int Ophthalmol. 1992;16(4-5):391-6.

21 Iwao K, Inatani M, Ogata-Iwao M, Takihara Y, Tanihara H. Restricted post-trabeculectomy bleb formation by conjunctival scarring. Graefes Arch Clin Exp Ophthalmol. 2009; 247(8):1095-101.

22 Miller KN, Blasini M, Shields MB, Ho CH. A comparison of total and partial tenonectomy with trabeculectomy. Am J Ophthalmol. 1991;111(3):323-6.

23 Dubey S, Singh N. Excision of the Tenon capsule in pediatric trabeculectomy: a controlled study. J Glaucoma. 2017;26(3):e126-e7.

24 Seet LF, Finger SN, Chu SW, Toh LZ, Wong TT. Novel insight into the inflammatory and cellular responses following experimental glaucoma surgery: a roadmap for inhibiting fibrosis. Curr Mol Med. 2013;13(6):911-28.

25 Qiu S, Jia Y, Sun Y, Han P, Xu J, Wen G, et al. Von Hippel-Lindau (VHL) protein antagonist VH298 improves wound healing in streptozotocin-induced hyperglycaemic rats by activating hypoxia-inducible factor- (HIF-) 1 signalling. J Diabetes Res. 2019;2019:1897174.

26 Sunkari VG, Lind F, Botusan IR, Kashif A, Liu ZJ, Ylä-Herttuala S, et al. Hyperbaric oxygen therapy activates hypoxia-inducible factor 1 (HIF-1), which contributes to improved wound healing in diabetic mice. Wound Repair Regen. 2015;23(1):98-103. 
27 Jing L, Li S, Li Q. Akt/hypoxia-inducible factor- $1 \alpha$ signaling deficiency compromises skin wound healing in a type 1 diabetes mouse model. Exp Ther Med. 2015;9(6):2141-6.

28 Duscher D, Maan ZN, Whittam AJ, Sorkin M, Hu MS, Walmsley GG, et al. Fibroblast-specific deletion of hypoxia inducible factor-1 critically impairs murine cutaneous neovascularization and wound healing. Plast Reconstr Surg. 2015;136(5):1004-13.

29 Zada M, Pattamatta U, White A. Modulation of fibroblasts in conjunctival wound healing. Ophthalmology. 2018;125(2):179-92.

30 Ahluwalia A, Tarnawski AS. Critical role of hypoxia sensor: HIF-1alpha in VEGF gene activation. Implications for angiogenesis and tissue injury healing. Curr Med Chem. 2012; 19(1):90-7.

31 Wilgus TA. Vascular endothelial growth factor and cutaneous scarring. Adv Wound Care. 2019;8(12):671-8.
32 Liu CJ, Huang YL, Chiu AW, Ju JP. Transcript expression of matrix metalloproteinases in the conjunctiva following glaucoma filtration surgery in rabbits. Ophthalmic Res. 2004; 36(2):114-9.

33 Wang L, Liu MY, Yin G, Li N, Wang DB. Exogenous tissue inhibitor of metalloproteinase- 2 affects matrix metalloproteinase- 2 expression in conjunctival filtering blebs and bleb scarring in rats. Biomed Res Int. 2018; 2018:9365950.

34 Klink T, Schrey S, Elsesser U, Klink J, Schlunck G, Grehn F. Interobserver variability of the Würzburg bleb classification score. Ophthalmologica. 2008;222(6):408-13.

35 Wells AP, Crowston JG, Marks J, Kirwan JF, Smith G, Clarke JC, et al. A pilot study of a system for grading of drainage blebs after glaucoma surgery. J Glaucoma. 2004;13(6): 454-60.

36 Cantor LB, Mantravadi A, WuDunn D, Swamynathan K, Cortes A. Morphologic classification of filtering blebs after glaucoma filtration surgery: the Indiana Bleb Appearance Grading Scale. J Glaucoma. 2003;12(3):26671.
37 Zhang Z, Nie F, Kang C, Chen B, Qin Z, Ma J, et al. Increased periostin expression affects the proliferation, collagen synthesis, migration and invasion of keloid fibroblasts under hypoxic conditions. Int J Mol Med. 2014; 34(1):253-61.

38 Valle-Tenney R, Rebolledo D, Acuna MJ, Brandan E. HIF-hypoxia signaling in skeletal muscle physiology and fibrosis. J Cell Commun Signal. 2020;14(2):147-58

39 Lin M, Hu Y, Chen Y, Zhou KK, Jin J, Zhu M, et al. Impacts of hypoxia-inducible factor-1 knockout in the retinal pigment epithelium on choroidal neovascularization. Invest Ophthalmol Vis Sci. 2012;53(10):6197-206.

40 Lin M, Chen Y, Jin J, Hu Y, Zhou KK, Zhu M, et al. Ischaemia-induced retinal neovascularisation and diabetic retinopathy in mice with conditional knockout of hypoxia-inducible factor-1 in retinal Müller cells. Diabetologia. 2011;54(6):1554-66. 